\title{
A Prediction Method of Wear for Volute Casing of a Centrifugal Slurry Pump
}

\author{
Baocheng Shi $(\mathbb{D}$, Jianpeng Pan, Lijuan Wu, Xingkai Zhang, Yijie Qiu, and Yindi Zhang \\ School of Petroleum Engineering, Yangtze University, Wuhan 430100, China \\ Correspondence should be addressed to Baocheng Shi; shibaoch@yangtzeu.edu.cn
}

Received 11 August 2020; Revised 18 September 2020; Accepted 19 November 2020; Published 18 December 2020

Academic Editor: Jinze Xu

Copyright (C) 2020 Baocheng Shi et al. This is an open access article distributed under the Creative Commons Attribution License, which permits unrestricted use, distribution, and reproduction in any medium, provided the original work is properly cited.

\begin{abstract}
Volute wall wear situations directly affect a long time safe operation for the centrifugal slurry pump unit and the whole system. In the present study, internal flow field is numerically investigated in a solid-liquid centrifugal pump, and the volute wall wear caused by the solid-liquid two-phase flow is predicted with wear equation. A systematic analysis on the wear mechanism of the centrifugal pump volute wall is carried out deeply, including the volute wall wear region, wear rate, and the relationship among inlet flow rate, particle concentration, and particle size. The predicted high erosion intensity area shows good agreement with the experimental erosion area, and the predicted and experimental areas are both located at the volute angle of $180^{\circ}$ and near tongue. Therefore, the wear equation put forward in the present study is effective for estimating the erosion intensity and predicting the erosion area around the volute casing of a centrifugal pump.
\end{abstract}

\section{Introduction}

When the two-phase centrifugal pump runs in a high speed rotation, the wear of the impeller and volute has become one of the important reasons causing the material damage or equipment failure $[1,2]$. The continuing impact force and the friction, which are caused by interparticle collisions and collisions between solid particles and impeller and volute, seriously affect the service life of pump, and the impeller and volute are the most vulnerable parts of wear and tear. The present study is mainly focused on developing a quantitative prediction method to investigate the wear of volute through numerical analysis. As we know, the research in the influences of the liquid-solid two-phase flow on volute wall wear in the solid-liquid two-phase centrifugal pump is still in their infancy due to the complex phenomena and factors of erosion wear. These factors interact with one another, and the change of one factor may create a chain reaction in the volute wall wear rate, resulting in the change of wear mechanism. There have been short of systematic analysis and comprehensive research on the wear mechanism of the centrifugal pump volute, including the volute wall wear region, wear rate, and the relationship among inlet velocity, particle concentration, and particle size. At present, most results are only from scene experience. However, volute wall wear conditions are very important, which influence the working state and the using of longevity of the centrifugal pump unit and the whole system. In the present study, internal flow field was numerically investigated in a solid-liquid centrifugal pump, and the volute wear caused by the solidliquid two-phase flow was predicted with wear equation. We analyzed the wall wear mechanism for different parts of volute by considering the influences of structural parameters, operation parameters, and particle properties on the volute wall wear.

Currently, in order to effectively control and reduce the loss caused by wear and to improve the service life of equipment and materials, researchers have extensively investigated solid-liquid two-phase flow rules inside the pump and wall wear mechanism caused by solid particles. Most of the studies are centered on the analysis of particle properties and trajectories by experiments and calculations during the process of wall collision wear under different flow parameters. Based on the analysis of boiler pipeline failure, Tilly [3] found that about a third wear was caused by abrasion. Liu [4] investigated numerically silt abrasive erosion of hydraulic machinery for 
low sand-water concentrations (mean sand concentration by volume $\left.\left(C_{\mathrm{v}}<1 \%\right)\right)$. In his study, the turbulent flow velocity fields, pressure distributions, and the trajectories of sand particles in the hydraulic machinery flow were numerically calculated using the mixed Eulerian-Lagrangian turbulence model. In addition, the sand particle rebound model and the erosion wear model in the hydraulic machinery flow field were also presented. Li and Han [5] simulated the flow field of the slurry pump by using a $k-\varepsilon$ two equations model and SMPLE algorithm. The simulation results suggested that the concentration of the solid phase and the wear in the blade and the volute increased with the increase of particle diameter and initial concentration of the solid phase. However, when the particle diameter and initial concentration of the solid phase were increased to a certain value, the concentration of the solid phase in the volute would keep unchanged. By comparison analysis, Shu, $\mathrm{Xu}$, and Tang [6] found that small particles were liable to impact the rear-end surface of impeller adjacent to the outlet side, while large solid particles could repeatedly impact the machine surface more easily than small particles. The numerical simulation results were verified by experimental results. An extensive parametric study has been carried out in order to optimize the shroudtype impeller taking into account the blade discharge angle, addition splitter blades, and modified blade (backward long blades) and find that the hydraulic efficiency of the centrifugal slurry pump can be increased up to $9 \%$ by using the backward long blades in addition to modified volute compared to the original ones $[7,8]$.

Because of the complexity of the material erosion behavior, there is no acceptable, universally applicable formula to predict erosion resistance of materials. So far, no reasonable explanation has been given for the material size effect or brittle-ductile transition, and the influences of particle trajectories on the material erosion behavior and particle size distribution on the plastic material erosion behavior are not clearly reported. Since the first erosion theory, micro cutting theory, several similar theories have been put forward to explain or predict the erosion behavior of materials [9-16]. Unfortunately, none of these theories can successfully reveal the inner mechanism of the material erosion. Tuzson [17] found that erosion damage accumulated over a considerable time period, and the material removal rate was found to be approximately proportional to the local rate of power dissipation. The erosion depth would then be proportional to the local energy dissipated by the slurry particles. Wilson, Addie, and Sellgren [18] believed that the removal of material over a time occurs through small-scale deformation, cutting, fatigue cracking, or a combination of these and thus depends on the properties of both the wearing surface and the particles. Ductile materials tend to exhibit erosion primarily by deformation and cutting, with the specific type depending on the angularity of the eroding particles. Brittle or hardened materials tend to exhibit fatigue cracking erosion under repeated particles impact. The useful life of the most slurry pump is limited by erosive wear of the flow passages. Sundstrom and Rendon [19] pointed out that particles with lower hardness than that of the abraded surface results in much lower wear rates than harder particles.
In the present study, a solid-liquid two-phase centrifugal pump is taken as the research object. Based on CFD (computational fluid dynamics), internal flow field is calculated, and the wear equation is employed to calculate the volute wear and wear area caused by the solid-liquid two-phase flow. On the basis of the above analysis, the wear mechanism of different parts of the volute is analyzed. The influences of structural parameters, operation parameters, and particle properties on the volute wall wear are analyzed, and the volute wall wear mechanism is obtained. These studies provide a theoretical and practical basis for the optimization design and safe operation of the solid-liquid two-phase centrifugal pump.

\section{Numerical Methods}

2.1. Governing Equations. For the two-phase flow centrifugal slurry pump at low solid phase volume fractions (usually no more than 10\%), the solid-liquid two-phase flow can be numerically simulated with the discrete phase model. The characteristics of the discrete phase model (DPM) are oneway interaction. The fluid, as a carrier, has influences on the movement of the particles by drag, drop, and vortex, while the solid particle effects on the liquid phase flow which is very limited and can be negligible.

As the continuum phase in the centrifugal slurry pump, water is incompressible, thus the Renault eddy viscosity model is employed to calculate the liquid flow field. The continuity equation, momentum equation, and RNG (renormalization group) $k-\varepsilon$ turbulence model equations can be referred to the literatures [20-24].

In numerical calculations, the solid particle movement can be described by Lagrange's equation. Based on a single particle forces analysis, the particle trajectory can be solved by integral of differential equations. According to Newton's second law, control equations for spherical particles in the slurry pump flow field in the $x$ direction in the absolute coordinates system can be given as follows:

$$
\frac{1}{6} \pi d_{p}^{3} \rho_{p}\left[F_{b}+F_{p}+F_{d}+F_{v}\right]=m_{p} \frac{d u_{p}}{d t}
$$

where $F_{b}$ is the buoyancy, $F_{b}=\left(\rho_{p}-\rho_{l} / \rho_{p}\right) g_{x i} ; F_{p}$ is the resistance caused by flow pressure gradient, $F_{p}=\left(\rho_{l} / \rho_{p}\right)$ $u_{p}\left(\partial u / \partial x_{i}\right) ; F_{d}$ is the drag, $F_{d}=\left(18 \mu / \rho_{p} d_{p}^{2}\right)\left(C_{d} \operatorname{Re}_{p} / 24\right)\left(u_{l}\right.$ $\left.-u_{p}\right) ; F_{v}$ is the additional mass force, $F_{v}=1 / 2\left(\rho_{l} / \rho_{p}\right)\left(d\left(u_{l}\right.\right.$ $\left.\left.-u_{p}\right) / d t\right) ; d_{p}, \rho_{p}, m_{p}$ and $u_{p}$ are the solid particle diameter, density, mass, and velocity, respectively, $\rho_{l}$ and $u_{l}$ are the density and velocity of liquid; $g_{x i}$ is the gravitational acceleration; $\mu$ is the dynamic viscosity of the liquid; $C_{d}$ is the drag coefficient; and $\mathrm{Re}_{p}$ is the particle Reynolds number.

In the present study, the slurry flow through the centrifugal pump was modeled using the DPM approach with a multiphase model in FLUENT ${ }^{\circledR}$. The RNG turbulence model with swirling flow correction was employed in the turbulent core region, and the wall function method was selected to treat the low Re flow near the solid wall. The SIMPLE algorithm was applied to deal with pressure-velocity coupling. 
TABLE 1: The basic design parameters of the impeller.

Rated flow Design head Specific speed Vane number Impeller outer diameter Impeller inlet diameter Blade inlet/outlet incidence $Q_{0}=15.1 \mathrm{~L} / \mathrm{s} \quad H=15.1 \mathrm{~m} \quad n_{s}=85 \quad Z=5 \quad D_{2}=228 \mathrm{~mm} \quad D_{0}=100 \mathrm{~mm} \quad \beta_{1}=15^{\circ}, \beta_{2}=30^{\circ}$

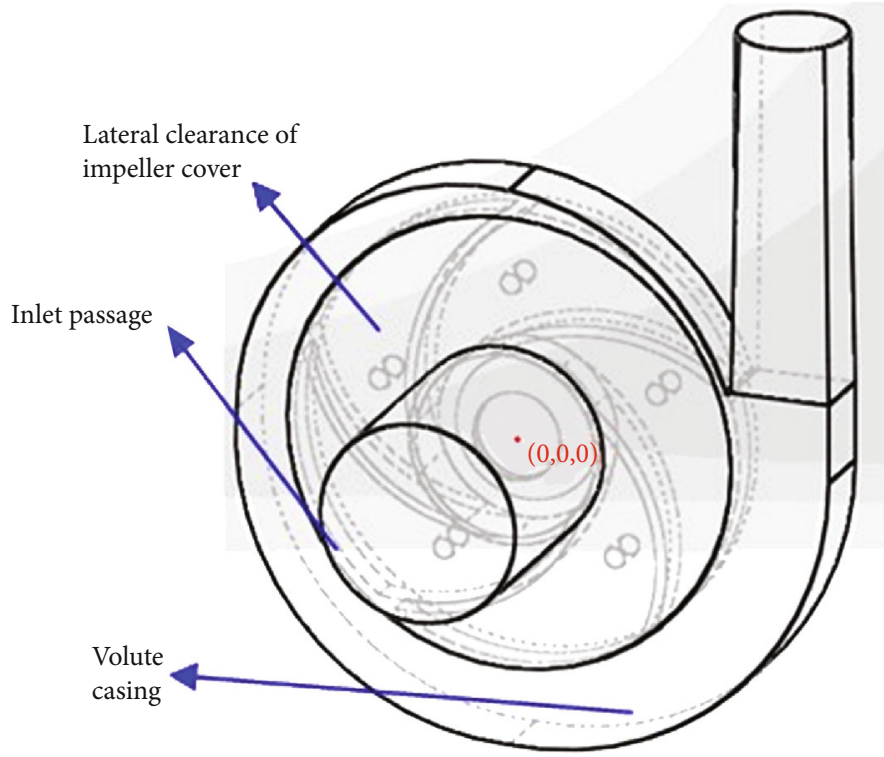

FIgURE 1: The whole flow field model (Shi et al. [21]).

Second order upwind difference scheme was used for the discretization of momentum, $k$, and $\varepsilon$ terms.

2.2. Prediction Model of Wear. Erosion models can be used to calculate the damage caused by particle impact on the wall. These models include Finnie [9], Oka [14, 15], and McLaury [25]. The default constant of the Finnie erosion model is only applicable to the erosion calculation of the carbon steel wall impacted by sand particles. McLaury [25] proposed a model for predicting the erosion rate of sand in water, which is mainly used to simulate the erosion rate in the process of mud erosion. The model constants and both need to be determined by experiments. The model constants given in the literature are only valid for water and sediment suspensions. The particle collision velocity is assumed to be in the range of $0-10 \mathrm{~m} / \mathrm{s}$. In actual flow, the solid particle velocity and collision angle are widely distributed, and the simulated particles come in a variety of shapes and sizes, so an ideal wear equation should be applied to different simulated calculation very well and should not have too many restrictions. The Oka wear equation $[14,15]$ is applicable to any hardness material, impact angle, and impact speed with a broader scope and relatively fewer restrictions than other wear equations, so it is selected in the present study. The Oka wear equation can be given as follows:

$$
\begin{aligned}
& E(\theta)=g(\theta) E_{90}, \\
& g(\theta)=(\sin \theta)^{n 1}\left[1+H_{v}(1-\sin \theta)\right]^{n 2},
\end{aligned}
$$

$$
\begin{aligned}
& E_{90}=K\left(H_{v}\right)^{k 1}\left(\frac{V_{p}}{V^{\prime}}\right)^{k 2}\left(\frac{D_{p}}{D^{\prime}}\right)^{k 3}, \\
& n 1=s 1\left(H_{v}\right)^{q 1}, n 2=s 2\left(H_{v}\right)^{q 2}, k 2=2.3\left(H_{v}\right)^{0.038} .
\end{aligned}
$$

Here, $E(\theta)$ is the wear loss in an arbitrary impact angle $(\mathrm{mmkg} / \mathrm{s}), E_{90}$ is the wear loss in a $90^{\circ}$ impact angle(mmkg/s), $H_{v}$ is the Vickers hardness(Gpa), $V_{p}$ and $V^{\prime}$ are the particle impact velocity and the reference impact velocity $(\mathrm{m} / \mathrm{s})$, and $D_{p}$ and $D^{\prime}$ are the particle diameter and the reference particle diameter $(\mu \mathrm{m})$.Other parameters are given as

$s 1=0.71, s 2=2.4, q 1=0.14, q 2=-0.94, K=65, k_{1}=-0.12, k_{3}=0.19$.

The wear rate is defined by

$$
R_{\text {erosion }}=\sum_{p=1}^{N_{\text {paticles }}} \frac{\dot{m}_{p} C\left(d_{p}\right) f(a) v^{b(v)}}{A_{\text {face }}}
$$

Here, $C\left(d_{p}\right)$ is a function of $d_{p}, f(a)$ is a function of the impact angle $a$, and $v$ is the impact velocity function. 


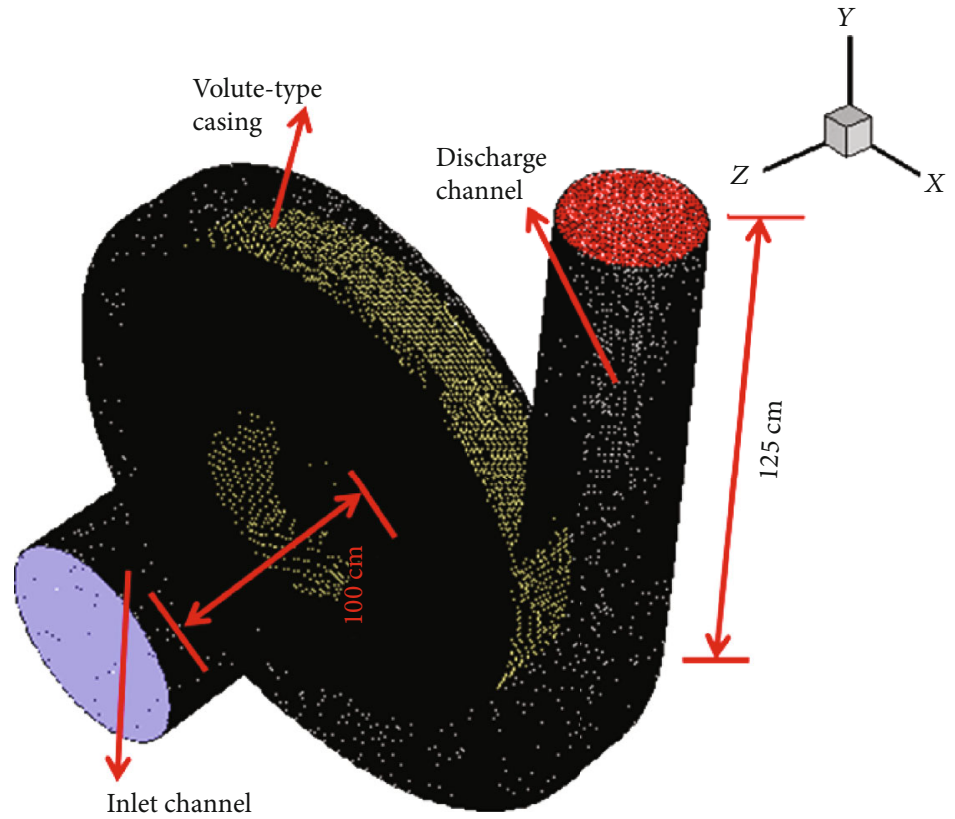

Figure 2: Mesh (Shi et al. [21]).

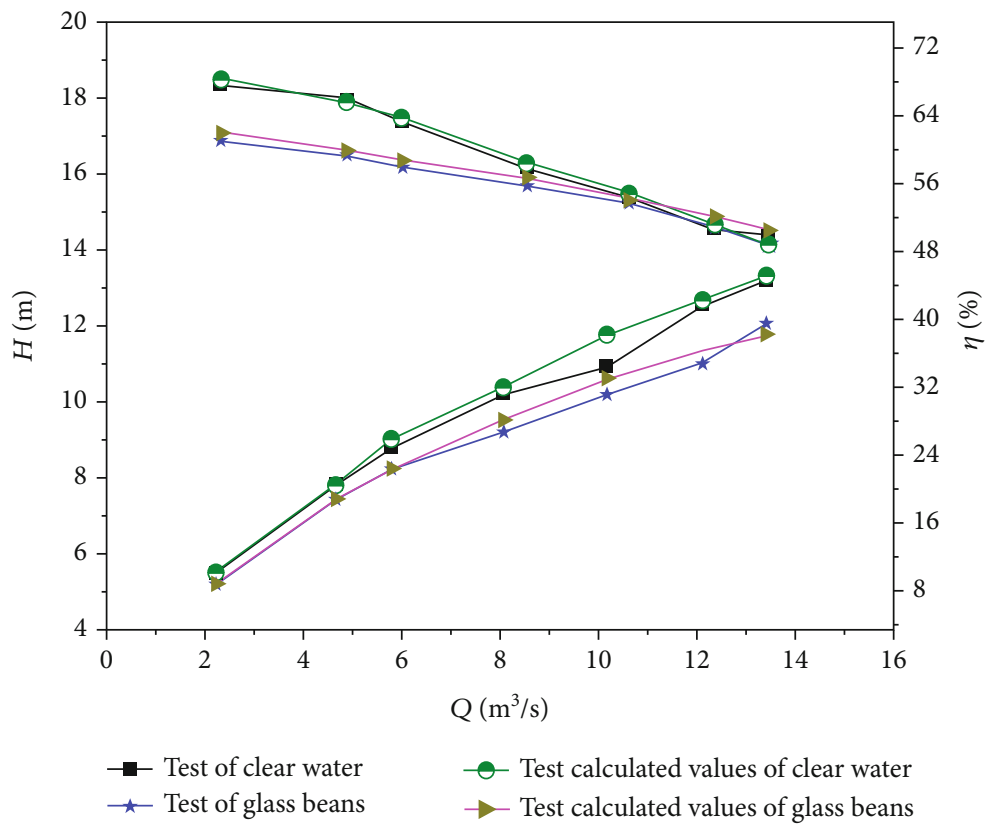

FIGURE 3: Comparison of the calculated performances with the experimental values.

Substitute the above wear equations (2)-(6) into wear rate equation (7):

$$
\begin{aligned}
C\left(d_{p}\right) & =\left(\frac{D_{p}}{D^{\prime}}\right)^{k 3} ; \\
f(\alpha) & =(\sin \alpha)^{n 1}\left[1+H_{v}(1-\sin \alpha)\right]^{n 2} E_{90} K\left(H_{v}\right)^{k 1} ; \\
v^{b(v)} & =\left(\frac{V_{p}}{V^{\prime}}\right)^{k 2} .
\end{aligned}
$$

Considering the unit size, a new wear rate equation can be given:

$$
R_{\text {erosion }}=\sum_{p=1}^{N_{\text {paticles }}} \frac{\dot{m}_{p} E(\alpha) 10^{-6}}{A_{\text {face }}} .
$$

Normal coefficients can be given:

$$
\varepsilon_{N}=0.993-0.0307 \alpha+4.75 \times 10^{-4} \alpha^{2}-2.61 \times 10^{-6} \alpha^{3} .
$$




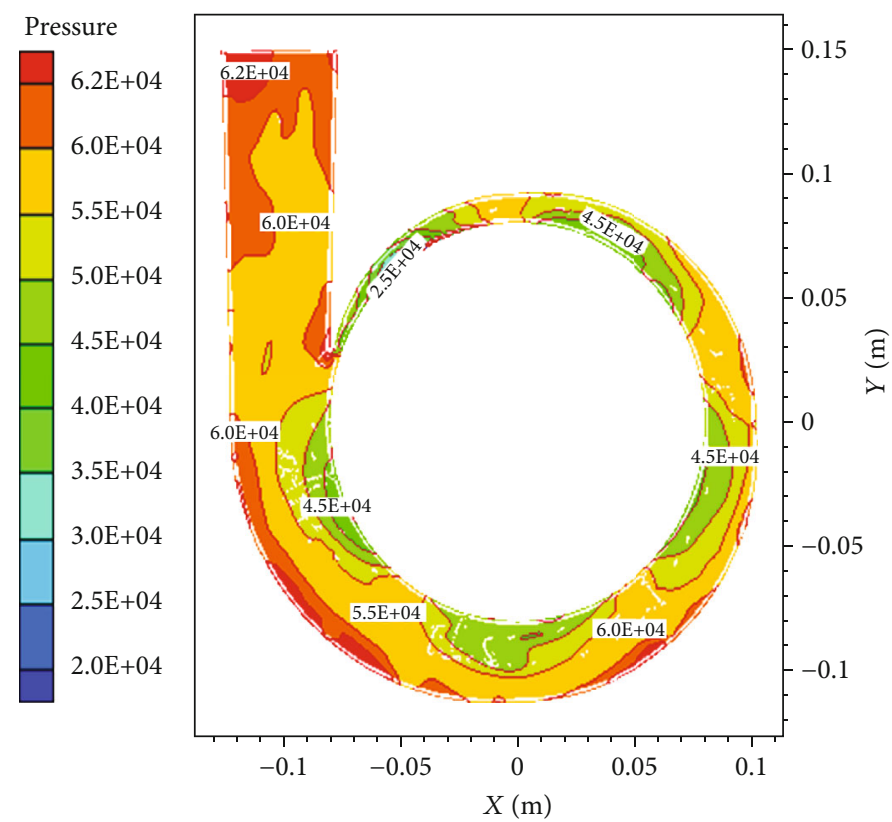

(a) Static pressure distribution for the liquid phase in center section (Pa)

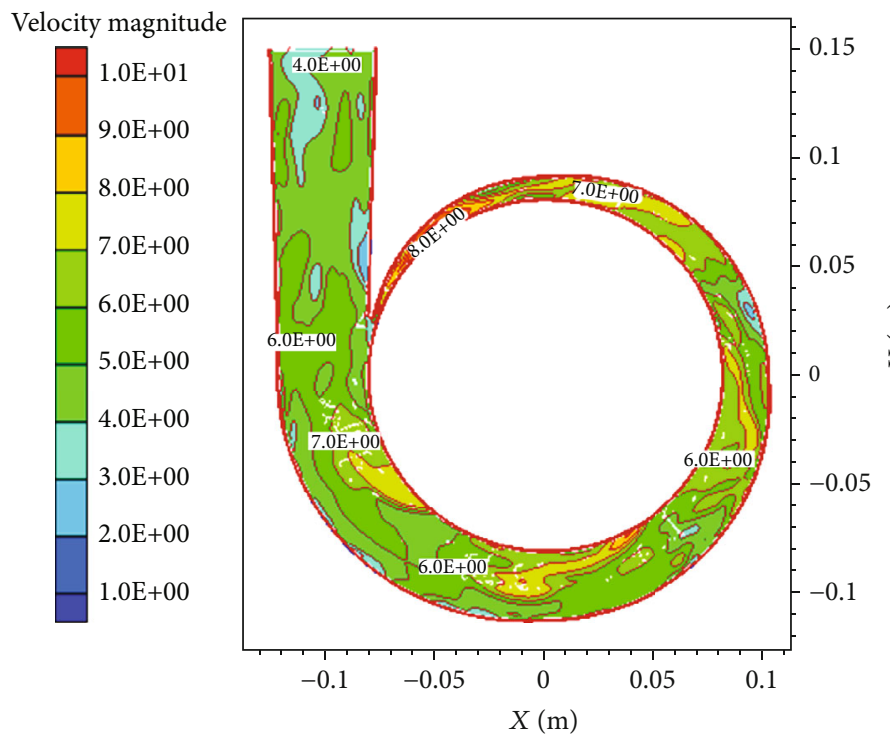

(b) Relative velocity distribution for the liquid phase in center section $(\mathrm{m} / \mathrm{s})$ dpm-erosion rate

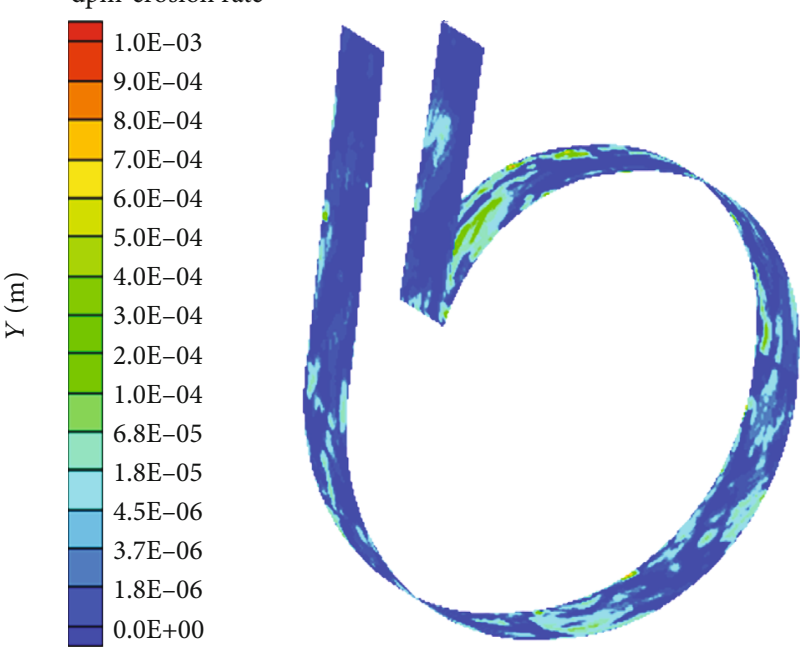

(c) Dpm-erosion rate distribution along the volute wall $\left(\mathrm{kgm}^{-2} \mathrm{~s}^{-1}\right)$

Figure 4: Continued. 


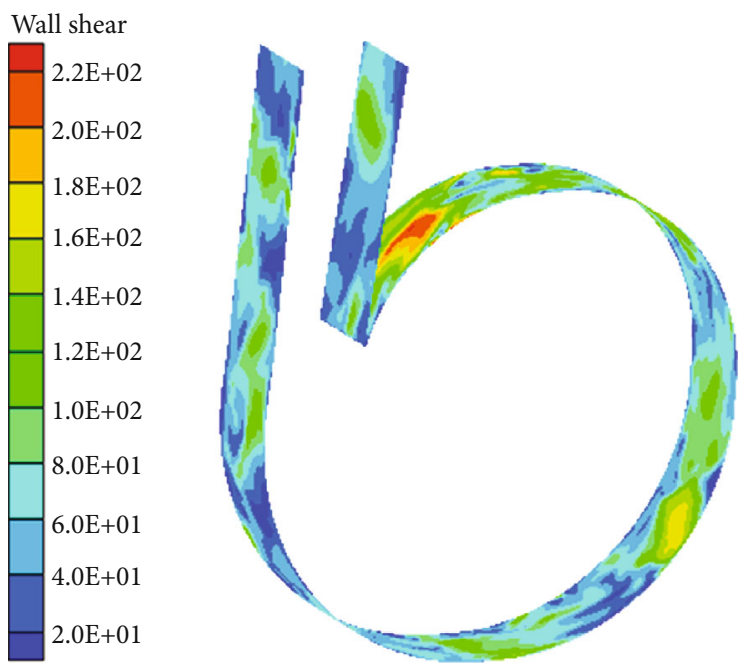

(d) The wall shear distribution along the volute wall $(\mathrm{Pa})$

Figure 4: Several important parameter distributions in the volute.

Tangent coefficients can be given:

$$
\varepsilon_{T}=0.998-0.029 \alpha+6.43 \times 10^{-4} \alpha^{2}-3.56 \times 10^{-6} \alpha^{3} .
$$

2.3. Pump Specifications and Calculation Conditions. We applied the above models to a centrifugal pump. The impeller of the pump has five blades and a volute-type casing, which is made of zinc. The maximum size of the impeller is $160 \mathrm{~mm}$, and the hub diameter is $56 \mathrm{~mm}$. Operating at the design point, the flow rate of the pump, $Q$, is $15 \mathrm{~m}^{3} / \mathrm{h}$, the head, $H$, is $20 \mathrm{~m}$, and the rotational speed is $1450 \mathrm{rpm}$. The main parameters of the impeller are listed in Table 1. The different flow channels (balance hole, lateral clearances of impeller cover and disk, impeller) of the pump model are shown in Figure 1. Figure 2 shows a schematic of the numerical mesh and boundary conditions used in the simulation. Figures 1 and 2 are reproduced from Baocheng and Shi et al., Numerical Simulation of 3D Solid-Liquid Turbulent Flow in a Low Specific Speed Centrifugal Pump: Flow Field Analysis. 2015. A cylindrical suction channel with an axial length of $100 \mathrm{~mm}$ is connected to the region including the impeller blades, and a $125 \mathrm{~mm}$ cylindrical discharge channel is also connected downstream of the volute-type casing. The total number of grids of the volutetype casing including the inlet channel, and the discharge channel is 812944 .

The liquid velocity at the inlet boundary keeps constant of $\mathrm{Q} / \mathrm{A}, \mathrm{m} / \mathrm{s}$. The solid particle radius is in the range of $30-$ $300 \mu \mathrm{m}$ at the inlet boundary. Nonslip conditions are assumed for the solid surface of the blade and shroud of the volute, and the assumption of nonslip conditions is also adopted for the wall surface of the suction and discharge channels.

To reduce the calculation time, the simulation process is divided into two stages and carried out sequentially. First, the flow field is formed using local time stepping [25]. The solid particles motion in this stage is assumed to remain in a quasiequilibrium status to avoid instability in calculation when the liquid phase pressure changes. Then, the solid-particle behavior can be calculated in detail, without assuming a qua-

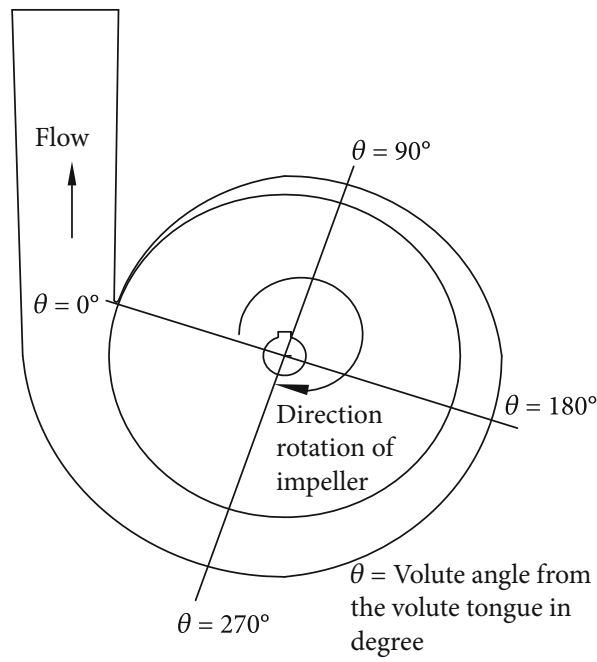

FIGURE 5: Schematic view of the volute casing with the location of volute angle.

siequilibrium status. The time step in the second stage of the simulation is 0.02 microseconds.

\section{Results and Discussion}

3.1. Pump Performance. The slurry pressure value at the solidliquid pump inlet section (i.e., inhalation tube) and total outlet pressure value at the water pressure chamber can be obtained by surface integral functions in the FLUENT ${ }^{\circledR}$. The pump head is defined as the total head difference between the inlet and outlet of the pump and can be expressed as

$$
H_{m}=\frac{P_{\text {mout }}-P_{\text {min }}}{\rho_{m} g}+\Delta z,
$$

where $H_{m}$ is the slurry pump head of the solid-liquid pump, $m, P_{\text {mout }}$ is the total pressure at the outlet section of the slurry 


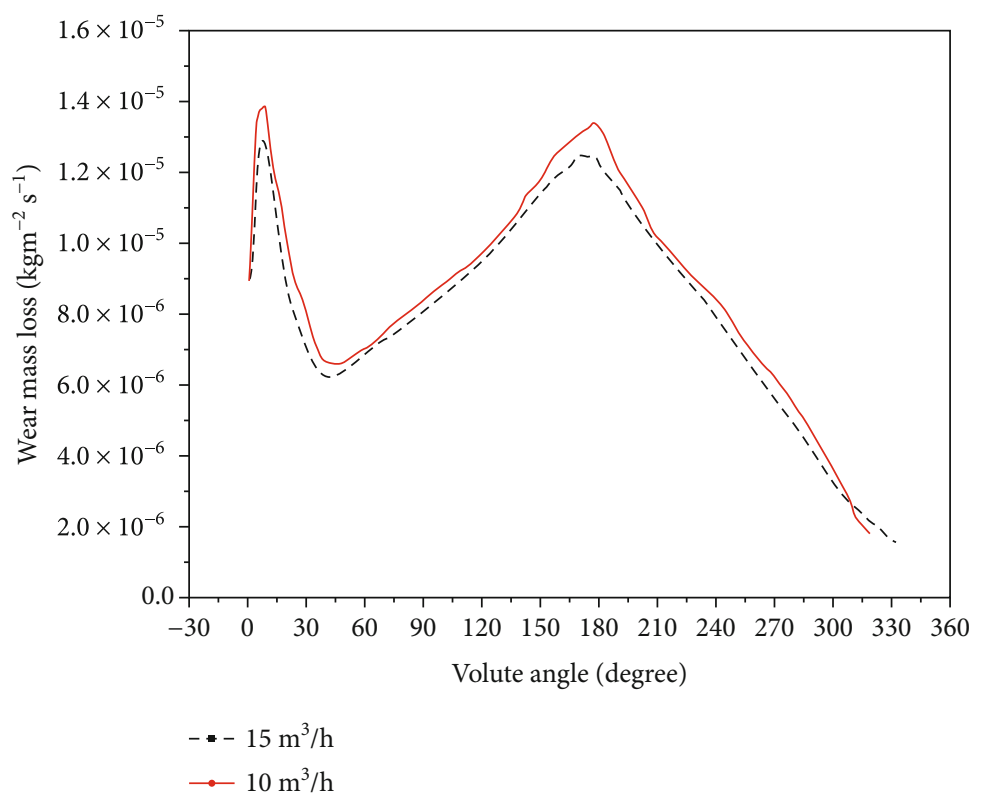

FIGURE 6: Variation in wear along the outer casing wall at two flow rates for the solid-liquid mixture of 5\%concentration (by volume).

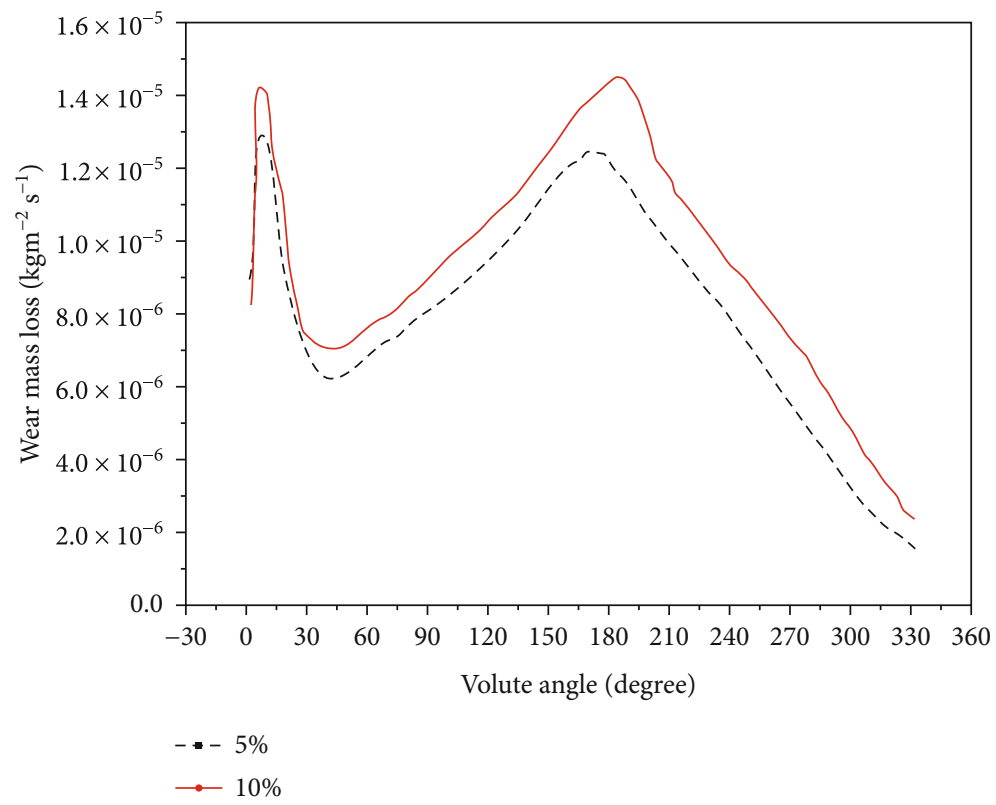

FIGURE 7: Variation in wear along the outer casing wall at two solid concentrations for the solid-liquid mixture at $15 \mathrm{~m}^{3} / \mathrm{h}$ discharge rate.

solid-liquid pump, $\mathrm{Pa}, P_{\min }$ is the total pressure at the inlet section of slurry solid-liquid pump, $\mathrm{Pa}$, and $\Delta z$ is the position head, namely, the difference of centre levels between the pump outlet passage and inlet passage in the vertical direction, $\mathrm{m}$. $P_{\text {mout }}$ and $P_{\text {min }}$ can be obtained by the surface integral function in FLUENT software, and slurry density $\rho_{m}$ and acceleration of gravity $g$ are known parameters, so the head of the solidliquid pump can be achieved conveniently and accurately by Eq.(12).

In order to improve the traditional efficiency prediction methods, based on the $3 \mathrm{D}$ numerical simulation of the whole flow field, a reasonable efficiency prediction formula is put forward to calculate the efficiency of solid-liquid pump as follows:

$$
\eta_{p}=\frac{\rho_{m} g Q H_{m}}{M \omega} \times 100 \% \text {, }
$$

where $\eta_{p}$ is the efficiency of the two-phase flow pump, $Q$ is the volume flow rate of the two-phase flow $\mathrm{m}^{3} / \mathrm{s}, M$ is the impeller torque, $\mathrm{N} \cdot m$, and $\omega$ is the impeller angular velocity, $\mathrm{rad} / \mathrm{s}$. Slurry density $\rho_{m}$, acceleration of gravity $g$, and impeller angular velocity $\omega$ are known parameters, $H_{m}$ can be obtained by 


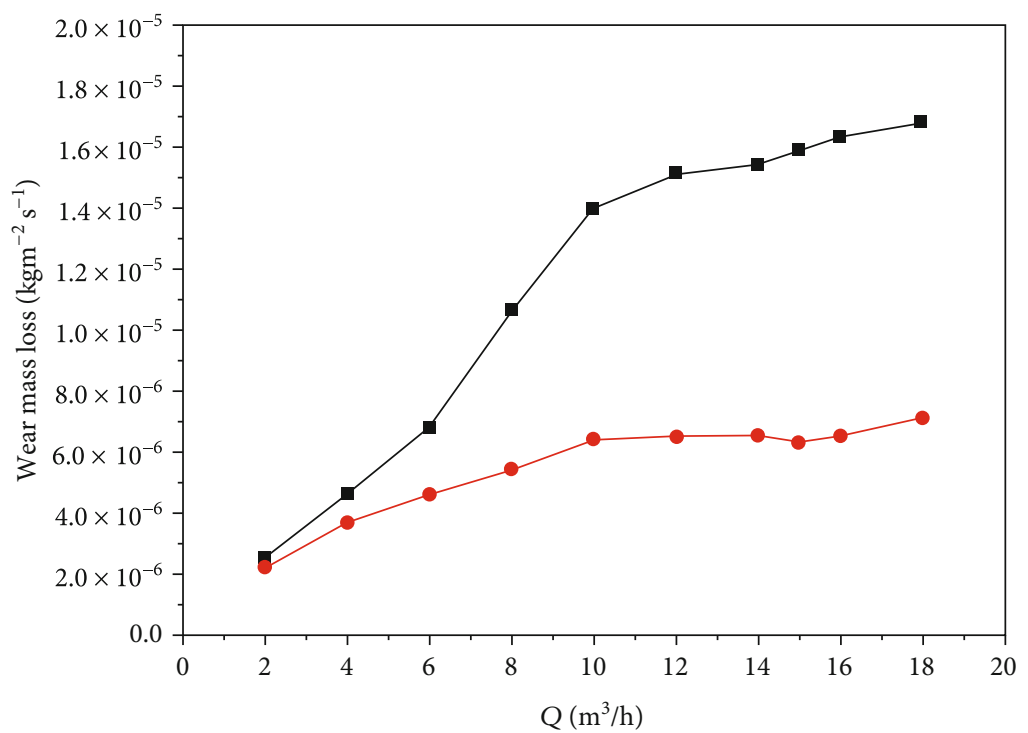

- The maximum values

- The average values

Figure 8: Variation of wear mass loss of volute material with the inlet flow rate.

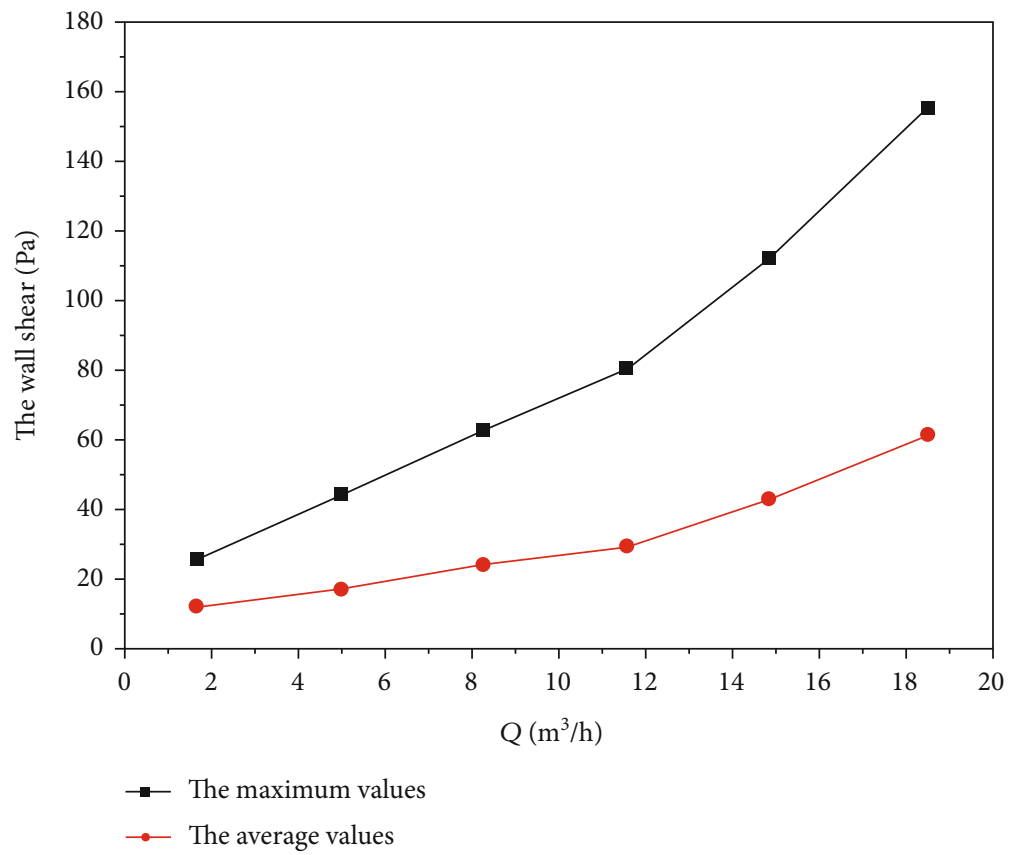

FIGURE 9: Variation of wall shear with the inlet flow rate.

Eq.(12), and impeller torque $M$ can be obtained from a forces report in FLUENT ${ }^{\circledR}$.

The comparisons of head and efficiency between the calculated results and experimental data under the rotational speed of $1450 \mathrm{rpm}$ are shown in Figure 3. From the comparison results of clear water and the two-phase flow with the solid-phase volumetric concentration of $10 \%$ and particle radius of $0.002 \mathrm{~mm}$, we find that the total head was equivalent to the pressure increment between the outlet of the suction pipe and the outlet of the volute, and the calculated results for clean water and the two-phase flow are close to the experimental data, with the head error less than $2 \%$ and efficiency error $4 \%$ at the design point. The results of comparison indicate that the method selected in the present study has good performance and reliability for particle-fluid twophase flows in the centrifugal slurry pump.

3.2. Static Pressure, Velocity, Dpm-Erosion Rate, and Wall Shear. Figure 4 shows the predictions for instantaneous distributions of static pressure, velocity for the liquid phase in the center section, the dpm-erosion rate, and the wear shear along the volute wall. The pressure of the fluid shows the 


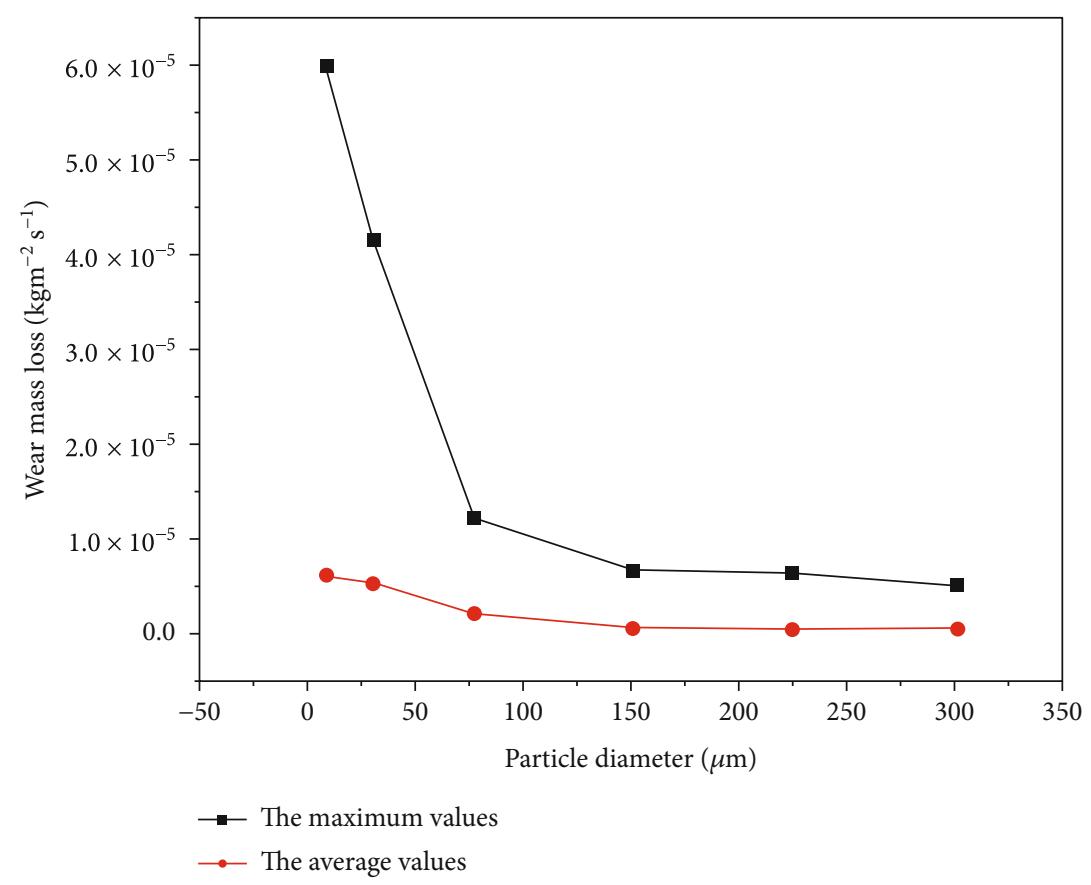

FIgURE 10: Variation of wear mass loss of volute material with the particle diameter.

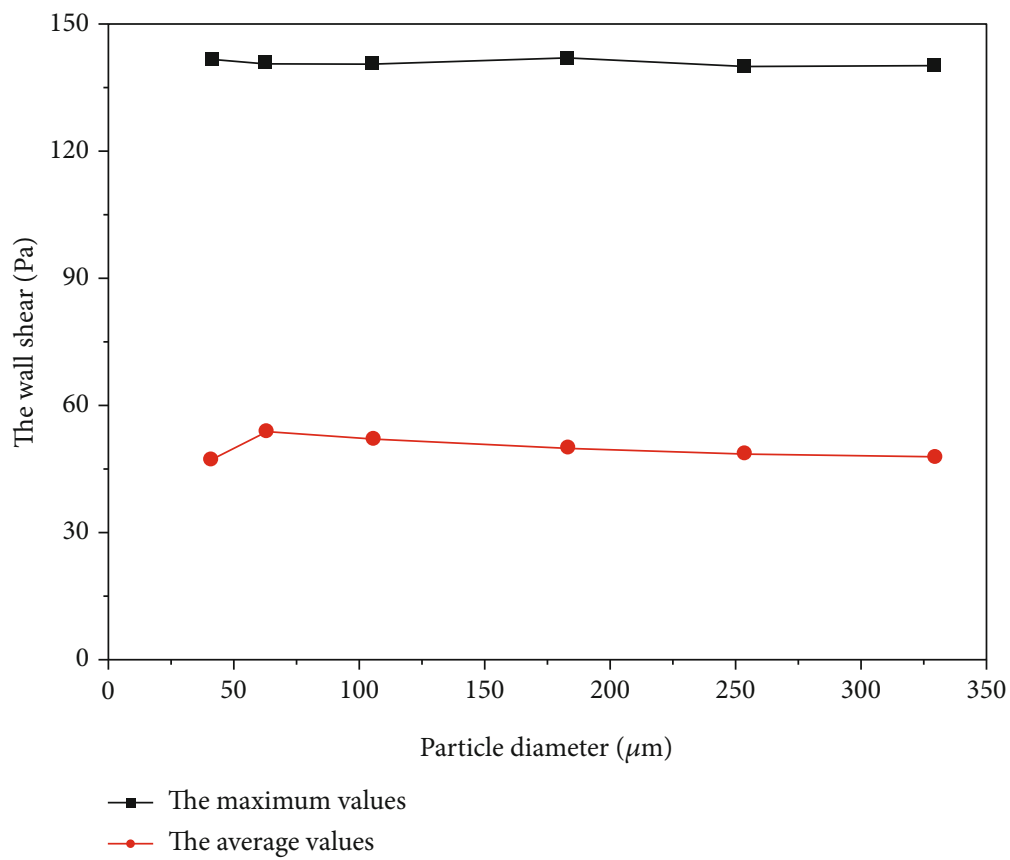

Figure 11: Variation of wall shear with the particle diameter.

trend of increasing from the impeller out to the volute wall, and the low-pressure region is formed near the tongue owing to the higher velocity here, as shown in Figure 4(a). Figure 4 (b) presents the velocity variations inside the volute for the liquid phase. The velocity distribution appears to be nonuniform, and the maximum values occur near the tongue. Two higher velocities occur at the volute angle of $30^{\circ}$ and $180^{\circ}$, respectively. Comparison of Figure 4(c) with Figure 4(d) shows that the dpm-erosion rate and the wall shear distributions along the volute wall are similar, and the maximum wall shear and erosion rate are both in the vicinity of the volute tongue and volute throat at $\theta=180^{\circ}$. This indicates that the impact angle of the solid particles decreases with increasing volute angle. Hence, it can be concluded that the variation in the impact angle of the solid particles is primarily responsible for the wear pattern observed at various operating conditions of the pump. But in the zone near to the volute tongue and volute throat, it is not feasible to identify the angle of impact, and these phenomena are also observed in the numerical prediction of trajectory for solid 


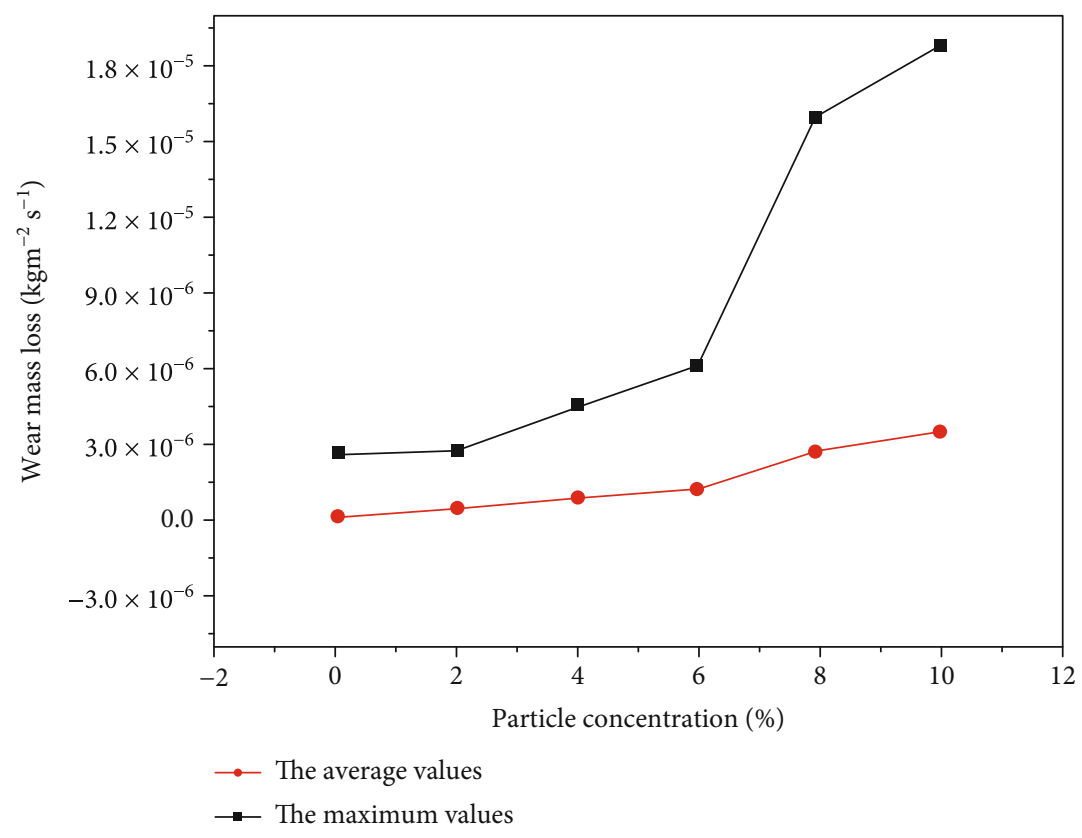

FIGURE 12: Variation of wear mass loss of volute material with particle concentration.

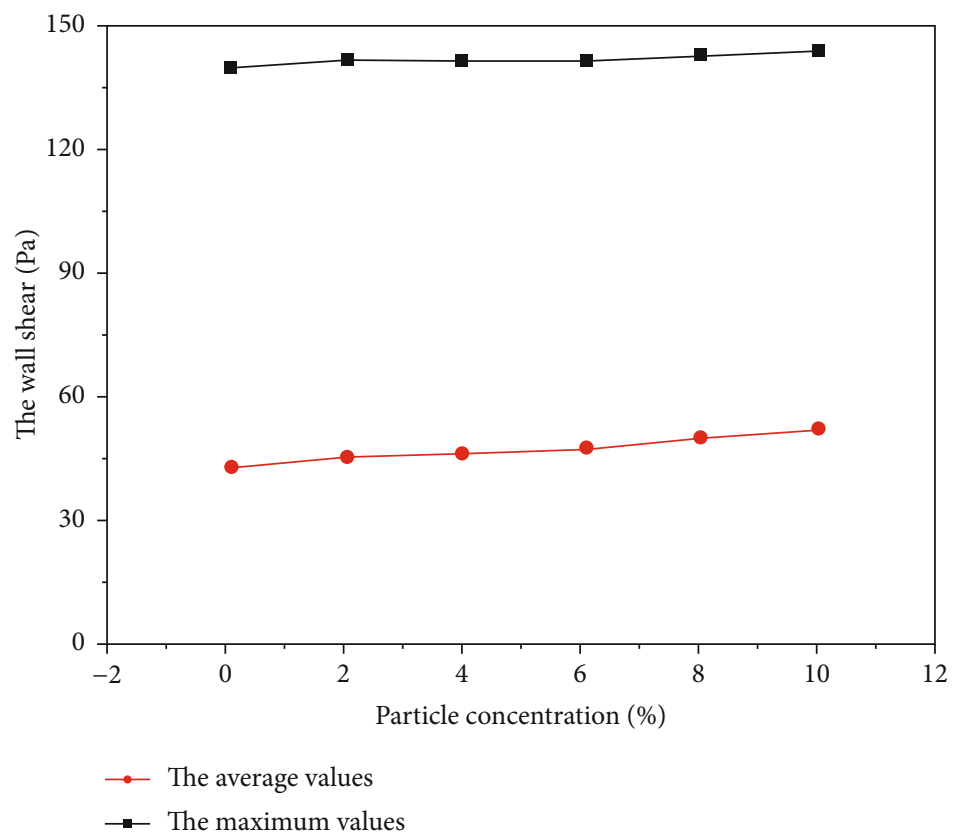

FIGURE 13: Variation of wall shear with particle concentration.

particles in a concentric-type casing by Minemura and Zhong [26].

3.3. Variation of Dpm-Erosion Rate with the Volute Angle. The variation of the dpm-erosion rate with the volute angle (as defined in Figure 5) is presented graphically in Figure 6 for a solid concentration of $5 \%$ (by volume) at two discharge rates, namely, $10 \mathrm{~m}^{3} / \mathrm{h}$ and $15 \mathrm{~m}^{3} / \mathrm{h}$. The discharge rate of 15 $\mathrm{m}^{3} / \mathrm{h}$ is the BEP (best efficiency point) flow rate for the assembled pump. At this discharge rate, we find that the $\mathrm{dpm}$-erosion rate decreases first and then increases with the increase of in the volute angle and attains the maximum value at $\theta=180^{\circ}$. The maximum wear around similar location for coatings of polyamide and epoxy resins on different casings with varying configurations (annular, semiannular, and quasispiral) was also observed in the prediction of Roco et al. [27], when operating the pump at the BEP flow rate. With further increase in the volute angle, the dpm-erosion rate decreases, and the rate of decrease is higher for angles beyond $180^{\circ}$. Similar observations based on the measurement of loss in wall thickness have been reported by Wiedenroth [28] for highly wear-resistant volute casing and by Roco 
et al. [27] for coatings of resin material at the volute surface. We also observed that the wear mass loss at the flow rate of $10 \mathrm{~m}^{3} / \mathrm{h}$ is a little higher than that at the BEP flow rate for the assembled pump. At lower flow rates, the flow velocities may be a little higher for volute angles less than $320^{\circ}$ due to higher recirculating flow in the volute, resulting in slightly higher wear at $10 \mathrm{~m}^{3} / \mathrm{h}$ discharge rate than that at $15 \mathrm{~m}^{3} / \mathrm{h}$ (105\% of BEP flow rate).

Figure 7 shows the trend of simulation curves of the $\mathrm{dpm}$-erosion rate with the volute angle for $5 \%$ and $10 \%$ solid concentration at $15 \mathrm{~m}^{3} / \mathrm{h}$ discharge rate. As shown in the figure, the variation and shape of the curve observed at $10 \%$ solid concentration appears similar to the one at $5 \%$. Compared to Figure 7, variation in wear along the outer casing wall at concentration of $5 \%$ and $10 \%$ is also similar to that at $8 \%$ concentration for the same discharge rate. Moreover, the dpm-erosion rate for $10 \%$ solid concentration is $10 \%$ $-40 \%$ higher than the corresponding values for $5 \%$ at the same discharge rate.

3.4. Influence of Particle Parameters on Wear Mass Loss. Figure 8 shows the volute wear mass loss of material changing with the inlet flow rate. It can be seen that the maximum wear values and the average wear values increase with the increase of the inlet flow rate. This indicates that the increasing velocity increases the probability of particle collision with the wall. Similar variation of wall shear with the inlet flow rate can be seen from Figure 9.

Figure 10 shows that the maximum volute wear mass loss of material and the average wear mass loss of material decrease with the increase of particle diameter. The maximum wear mass loss of material curves shows an obvious downward trend with the particle diameter when the particle diameters are less than $150 \mu \mathrm{m}$. This result indicates that the increasing solid particle diameter reduces the particle collision frequency with wall, and the particle collision energy is not the only reason for the mass loss of volute material. The variations of wall shear with the particle diameter and particle concentration are shown in Figures 11 and 12, respectively. The wall shear changes a little with variation of the particle diameter and particle concentration. Therefore, it can be included that the two parameters have limited influence on the wall shear.

The variation of wear mass loss of volute material with particle concentration is shown in Figure 13. It can be seen from the figure that the maximum wear values and the average wear values are both increasing with the increase of particle concentration. This indicates that the increasing velocity increases the probability of particle collision with the wall. The maximum wear curve is in an increasing trend. The curve changes a little when the concentration is less than $6 \%$, while the change is relatively large with further increasing the concentration. This indicates that the increasing particle concentration increases the probability of particle collision with the wall and enhances the erosion and abrasion of the volute wall.

\section{Conclusions}

Slurry pumps are extensively used in the hydraulic transportation of the solid-liquid mixture through pipes in various fields. For proper design and selection of a slurry pump, extensive data are required for accurate estimation of the pump performance for the case of the slurry flow at high concentrations. Based on the present investigations, it is concluded that the relative wear along the casing is a function of the erosion behavior of the volute casing material and variation of impact angles of solid particle around the casing. It is seen that the erosion is less at flow rates close to BEP than that at lower flow rates. The high erosion intensity areas predicted by the method are distributed in the vicinity of a volute angle of around $180^{\circ}$ and near the volute tongue. The results can provide a theoretical and practical reference for the two-phase flow pump optimization design to get a long period of safe operation.

\section{Data Availability}

The data used to support the findings of this study are included within the article.

\section{Conflicts of Interest}

The author(s) declare(s) that they have no conflicts of interest.

\section{Acknowledgments}

The authors gratefully expressed their thanks for the financial support by the National Natural Science Foundation of China (No. 51974033) and Educational Commission of Hubei Province of China (D20171305).

\section{References}

[1] G. Dong and J. Zhang, "Developments of research on the solid particle erosion of materials," Journal of Materials Science and Engineering, vol. 21, no. 2, pp. 307-312, 2003.

[2] Y. Ma, J. Ren, and Y. Li, "Development of research on erosion of materials," Journal of Lanzhou University of Technology, vol. 31, no. 1, pp. 21-25, 2005.

[3] G. P. Tilly, "Erosion caused by impact of solid particles," in Wear, vol. 13 of Treatise on Materials Science \& Technology, , pp. 287-319, Elsevier, 1979.

[4] X. B. Liu, "Numerical simulation of silt abrasive erosion in hydraulic machinery," Journal of Sichuan University of Science and Technology, vol. 19, no. 2, pp. 79-84, 2000.

[5] R. Li, Z. Xu, and W. Han, "Numerical simulation of interior flow and wear character in slurry pumps," Drainage and Irrigation Machinery, vol. 2, no. 25, pp. 5-11, 2007.

[6] L. J. Shu, H. Y. Xu, and L. L. Tang, "Numerical simulation of erosion and particle motion trajectory in centrifugal pump," Transactions of the Chinese Society for Agricultural Machinery, vol. 6, 2008.

[7] M. S. Cellek and T. Engin, "3-D numerical investigation and optimization of centrifugal slurry pump using computational fluid dynamics," Thermal Science and Technology, vol. 36, no. 1, pp. 69-83, 2016.

[8] M. S. Cellek and T. Engin, "Parametric investigation of a centrifugal slurry pump while handling clear water," Journal of Thermal Science \& Technology, vol. 36, no. 2, 2016. 
[9] I. Finnie, "The mechanism of erosion of ductile metals," $3 r d$ US National Congress of Applied Mechanics, vol. 25, pp. 527532, 1958.

[10] J. G. A. Bitter, "A study of erosion phenomena part I," Wear, vol. 6, no. 1, pp. 5-21, 1963.

[11] X. Zhang, Z. Jiang, R. Liao et al., "Study on temperature distribution of perforated horizontal wellbore," Journal of Thermal Science, vol. 29, no. 1, pp. 194-205, 2020.

[12] A. V. Levy, "The solid particle erosion behavior of steel as a function of microstructure," Wear, vol. 68, no. 3, pp. 269287, 1981.

[13] I. M. Hutchings, “A model for the erosion of metals by spherical particles at normal incidence," Wear, vol. 70, no. 3, pp. 269-281, 1981.

[14] Y. I. Oka, K. Okamura, and T. Yoshida, "Practical estimation of erosion damage caused by solid particle impact," Wear, vol. 259, no. 1-6, pp. 95-101, 2005.

[15] Y. I. Oka and T. Yoshida, "Practical estimation of erosion damage caused by solid particle impact," Wear, vol. 259, no. 1-6, pp. 102-109, 2005.

[16] P. Buffiere and R. Moletta, "Collision frequency and collisional particle pressure in three-phase fluidized beds," Chemical Engineering Science, vol. 55, no. 22, pp. 5555-5563, 2000.

[17] J. Tuzson, Centrifugal Pump Design, Wiley, New York, NY, 2000.

[18] K. C. Wilson, G. R. Addie, and A. Sellgren, Slurry Transport Using Centrifugal Pumps, Springer Science \& Business Media, 2006.

[19] A. Sundstrom, J. Rendon, and M. Olsson, "Wear behaviour of some low alloyed steels under combined impact/abrasion contact conditions," Wear, vol. 250, no. 1-12, pp. 744-754, 2001.

[20] B. Wu, H. Yan, and J. Zhang, "Numerical simulation and performance study on 3-D turbulence forecast of slurry pump," China Mechanical Engineering, vol. 20, no. 5, pp. 585-589, 2009.

[21] B. Shi and J. Wei, "Numerical simulation of 3D solid-liquid turbulent flow in a low specific speed centrifugal pump: flow field analysis," Advances in Mechanical Engineering, vol. 6, Article ID 814108, 2014.

[22] X. Zhang, D. Wang, R. Liao, S. Wang, B. Shi, and L. Wu, "Associated petroleum gas measurement at low gas content using PIS method," Flow Measurement and Instrumentation, vol. 70, p. 101662, 2019.

[23] X. Zhang, D. Wang, R. Liao, H. Zhao, and B. Shi, "Study of mechanical choked Venturi nozzles used for liquid flow controlling," Flow Measurement and Instrumentation, vol. 65, pp. 158-165, 2019.

[24] Q. Shen, Y. Jiang, F. Xia et al., "Hydrogen production by cobased bimetallic nano-catalysts and their performance in methane steam reforming," Petroleum Science and Technology, vol. 38, no. 6, pp. 618-625, 2020.

[25] B. S. McLaury, S. A. Shirazi, J. R. Shadley, and E. F. Rybicki, "Modeling erosion in chokes," in American Society of Mechanical Engineers, Fluids Engineering Division (Publication) FED, Vol. 236, pp. 773-781, ASME, 1996.

[26] K. Minemura, Y. Zhong, and T. Uchiyama, "Numerical Prediction of Erosion Wear on Pump Casing under Solid-Water Two-Phase Flow," Multiphase Flow, vol. 61, pp. 561-572, 1995.
[27] M. C. Roco, G. R. Addie, J. Dennis, and P. Nair, "Modeling erosion wear in centrifugal slurry pumps," Hydrotransport, vol. 9, 1984.

[28] W. Wiedenroth, "Evaluation of wear distribution of a dismountable impeller in a model dredge-pump," Fuel and Energy Abstracts, vol. 5, no. 38, pp. 358-364, 1997. 